[Technical Paper]

\title{
Failure Analysis and Stress Evaluation of Maleimide Molding Films during Temperature Cycling
}

\author{
Kazuhiro Kikuchi ${ }^{1,2}$, Kazue Uemura ${ }^{2}$, and Tadashi Suetsugu ${ }^{1 *}$ \\ ${ }^{1}$ Faculty of Engineering, Fukuoka University, 8-19-1, Nanakuma, Jonan-ku, Fukuoka-shi, Fukuoka 814-0180, Japan \\ ${ }^{2}$ R\&D Division, Lintec Corporation, 7-7-3, Tsuji, Minami-ku, Saitama-shi, Saitama 336-0026, Japan
}

(Received July 15, 2021; accepted October 12, 2021, published November 2, 2021)

\begin{abstract}
It is of paramount importance to develop semiconductor packaging materials with both high heat resistance and toughness. Thus, we designed and studied various maleimide molding sheets and investigated the relationship between the amount of flexible maleimide resin and defect development, including delamination and resin cracks, during temperature cycling (TC). The stress singularity parameters computed from the interfacial stress field during the TC and die shear tests showed that delamination became more likely as the amount of flexible maleimide increased. The flexural stress criterion for crack prevention was computed using stress singularity theory. Crack formations were observed for the tested packages of the materials with measured flexural stress values below the stress values of the criterion for the TC test. Furthermore, a simple equation was proposed to determine the stress criterion from material properties.
\end{abstract}

Keywords: Maleimide, Molding Sheets, Power Modules, Finite Element Analysis, Stress Singularity, Reliability, Heat Resistance, Flexural Toughness

\section{Introduction}

Wide-bandgap (WBG) semiconductors such as $\mathrm{SiC}$, $\mathrm{GaN}$, and GaAs have been increasingly adopted in power modules recently. WBG semiconductors provide higher efficiency than Si-based counterparts, reducing energy consumption, achieving environmental protection, and providing enhanced performance along with compactness. $[1,2]$ Although small power modules have high heat density due to switching losses, WBG semiconductors provide excellent heat resistance, enabling operation at temperatures exceeding $200^{\circ} \mathrm{C}$. Several methods have been proposed to manufacture power modules using fan-out panellevel packages and embedded die technology for integrated circuit packaging technologies.[3-6] Sheetmolding materials were applied to the methods because sheet-molding materials obviate the need for large molding equipment and expensive molds.[7] However, since molding materials with high heat resistance are required for WBG power modules, a glass-fiber-reinforced composite was proposed as one of the materials. [8, 9] Although the composites facilitate the control of panel warpage due

*: Corresponding author

E-mail: suetsugu@fukuoka-u.ac.jp to the low coefficient of thermal expansion (CTE), they suffer from low fluidity due to the small amount of resin. Furthermore, since the composite was conducted to reliability tests with maximum of $175^{\circ} \mathrm{C}$, no sheet-type encapsulating material with heat resistance of $200^{\circ} \mathrm{C}$ had been proposed.

Therefore, we previously reported a highly heat-resistant sheet-molding material using maleimide resin curing. $[10,11]$ However, the cured formulation was brittle. Thus, we investigated a new formulation providing both high heat resistance and toughness by blending two maleimide resins with relatively rigid (MA) and flexible (MB) structures, as shown in Fig. 1.[12] As a result, the cured formulation demonstrated increased flexural stress by blending $\mathrm{MB}$ and high heat resistance with a 5\% weight loss temperature of over $440^{\circ} \mathrm{C}$ and high adhesion properties to copper even after $1,000 \mathrm{~h}$ heat storage at $200^{\circ} \mathrm{C}$. However, when the blended MB exceeded a certain amount, resin cracks with delamination were generated, starting from the $\mathrm{Si}$ chip corner in the package-level temperature cycling (TC) test. The leading cause of crack formation was the CTE mismatch between the Si chip and the formulation because the CTE of the formulation increased with 
increasing amount of MB. Meanwhile, the flexural stresses of the formulations also increased with the amount of MB, but the relationships between these characteristics and defect development have not been clarified.

In this study, we studied the relationships between the crack development and the physical properties of maleimide molding sheets during the TC test. In general, cracking of molding material is caused by the extension of delamination. Therefore, we studied the interfaces prone to delamination by 2D-FEM (Finite Element Method) analysis of the package stress field under the TC test. Then, the stress field needed for interfacial delamination was estimated by FEM analysis of the die shear test. The relationship between the amount of $\mathrm{MB}$ in the maleimide molding sheet and interfacial delamination was discussed in terms of stress singularity parameters of their stress fields. Moreover, we computed the criterion of flexural stress in the evaluation package structure required to prevent crack propagation by applying stress singularity theory to the stress distribution in the package obtained by FEM analysis. Additionally, we proposed a simple equation to determine the stress criterion from the elastic modulus and CTE of the molding material.

\section{Evaluation Formulations and Packaging}

Maleimide molding sheets are comprised maleimide resins, a curing agent, a binder resin, coupling agents, and
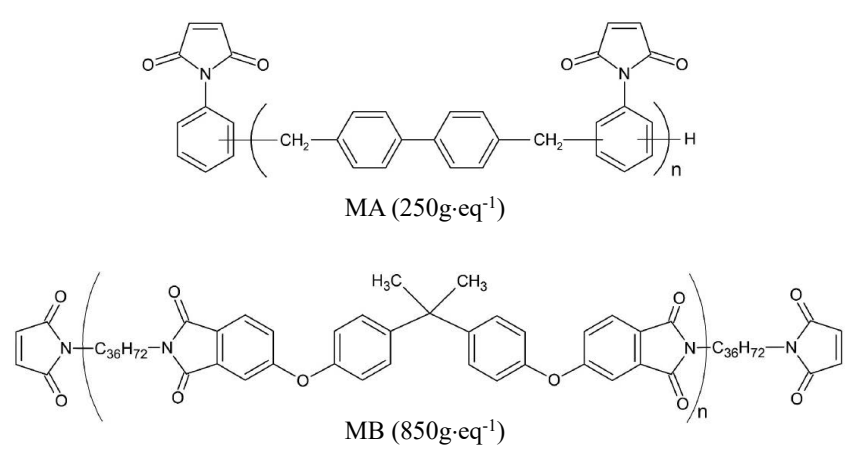

Fig. 1 Chemical structures and equivalent weights of the maleimide groups of relatively rigid maleimide resin (MA) and flexible maleimide resin (MB). silica particles as a filler. Keeping the weight ratio of these materials constant, we prepared formulations with different weight ratios of two types of maleimide resins: a rigid molecular structure possessing a biphenyl skeleton (MA) and a flexible molecular structure possessing long-chain alkyls (MB), shown in Fig. 1. Table 1 lists the blending ratios of the formulations and their properties. As a caveat, storage moduli were different from those we reported previously. These values were obtained from repeated measurements and accurately reflect the physical properties since the elastic modulus decreases as the flexible molecular structure increases. Therefore, the repeatedly measured data was applied in this work.

These formulations were used to fabricate packages for reliability evaluation. Figure 2 presents the structure of the fabricated package. The packaging process was performed as follows: Si chips were temporarily bonded to the cavity-formed PCB core using a single-sided adhesive tape, molded with the evaluation formulation on one side at a time, and then cured at $200^{\circ} \mathrm{C}$ for 4 h.[13, 14] Table 2 shows the package-level TC test results for each formulation. The TC test was conducted under $-55^{\circ} \mathrm{C} / 200^{\circ} \mathrm{C}$ with an exposure time of $10 \mathrm{~min}$ at each temperature up to

(a)

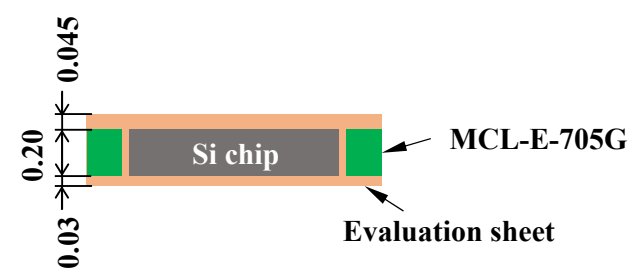

(b)

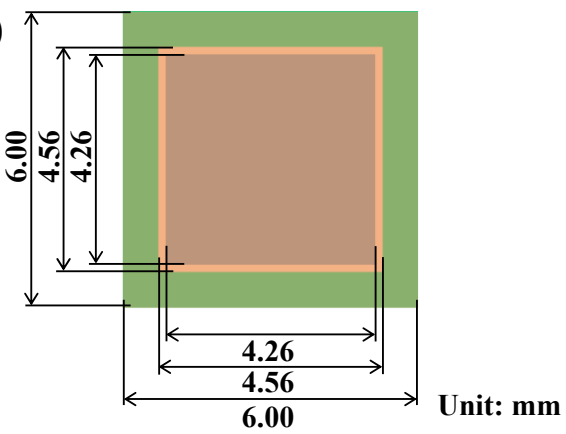

Fig. 2 Structure of the package used in the TC test. (a) Cross-sectional view, (b) Top view.

Table 1 Evaluation formulations and their properties

\begin{tabular}{ccccccc}
\hline Property & MMC1 & MMC2 & MMC3 & MMC4 & Silicon & Core \\
\hline Weight ratio (MA : MB) & $100: 0$ & $70: 30$ & $50: 50$ & $30: 70$ & - & - \\
Storage modulus (GPa) & 11.6 & 10.8 & 10.1 & 9.39 & 160 & 19.6 \\
CTE (ppm/K) & 23 & 38 & 45 & 58 & 3 & $6 / 13^{*}$ \\
Poisson's ratio & 0.3 & 0.3 & 0.3 & 0.3 & 0.26 & 0.3 \\
\hline
\end{tabular}

* Planer direction/Thickness direction 
Table 2 Results of the package-level TC test

\begin{tabular}{cccc}
\hline \multirow{2}{*}{ Formulation } & \multicolumn{2}{c}{$\begin{array}{c}\text { Number of non-defective packages/ } \\
\text { Number of inspections }\end{array}$} \\
& $\mathbf{3 0 0}$ cycle & $\mathbf{5 0 0}$ cycle & $\mathbf{1 , 0 0 0}$ cycle \\
\hline MMC1 & $12 / 12$ & $12 / 12$ & $12 / 12$ \\
MMC2 & $12 / 12$ & $12 / 12$ & $12 / 12$ \\
MMC3 & $12 / 12$ & $12 / 12$ & $12 / 12^{*}$ \\
MMC4 & $12 / 12$ & $10 / 12$ & $0 / 12$ \\
\hline
\end{tabular}

* White discoloration on the corner of Si chip

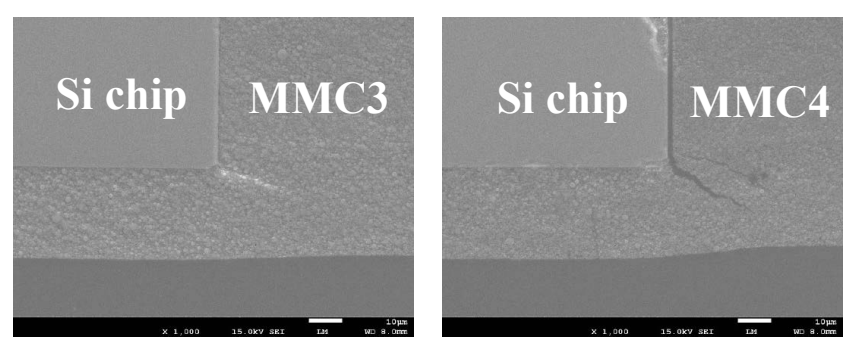

Fig. 3 Cross-sectional SEM image of the MMC4 package after the TC test for 1,000 cycles.

1,000 cycles. The evaluation criteria were determined based on results of visual inspections, interface failures analyzed by a scanning acoustic tomography, and crosssectional observation of tested packages. Although MMC1 and MMC2 passed 1,000 cycles, MMC3 and MMC4 developed defects in some packages after 1,000 and 500 cycles, respectively. Figure 3 shows the crack defects in MMC3 and MMC4 packages after 1,000 cycles. In particular, MMC4 developed cracks in all evaluation packages after 1,000 cycles, starting from the Si chip corner with delamination between the Si chip and the formulation.

\section{Interfacial Stress and Adhesive Strength \\ 3.1 Interfacial stress field in TC}

Since semiconductor packages comprise many types of materials, high interfacial stress is generated upon temperature changes due to the CTE mismatch of the individual components. This stress may cause defects to form at or near the interface, propagating and developing into cracks.[15-17] Here, the delamination mechanism was investigated by the FEM analysis of the interfacial stress around the Si chip corner, where the defects occurred in the TC test. Figure 4 presents the FEM model and analyzed location. The analytical model consisted of the right half of the package for symmetry structure, and the horizontal displacement at the center of the package was constrained. The FEM element type was chosen to be a four-

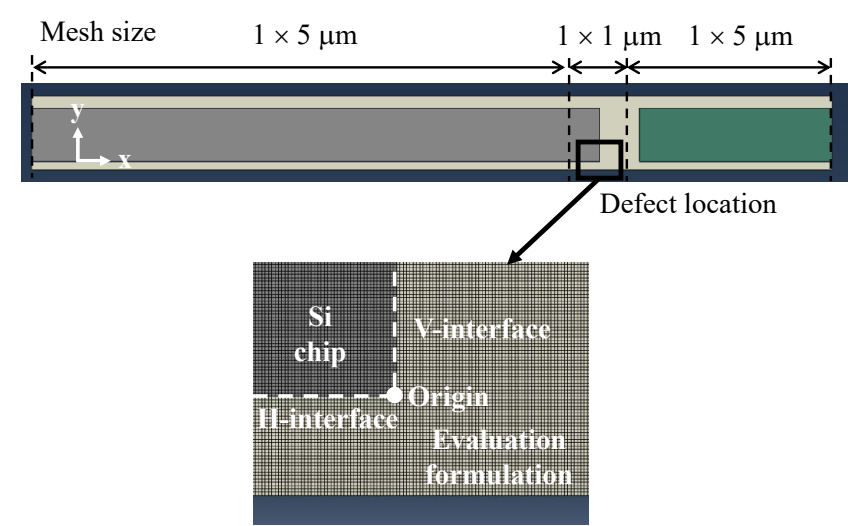

Fig. 4 FEM model of the package and interfacial stress analysis.

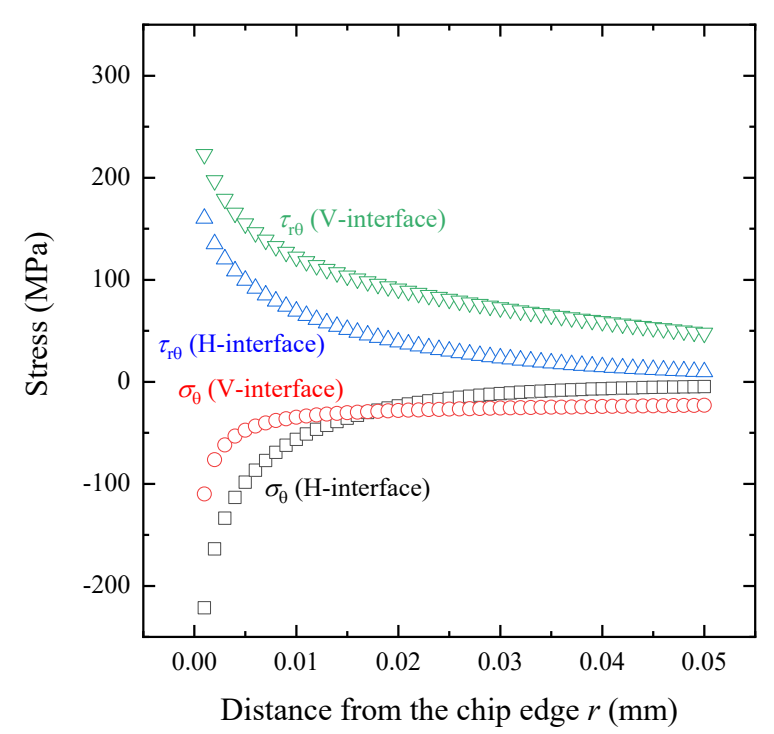

Fig. 5 Interfacial stress field at the low-temperature side of the temperature cycling test.

node two-dimensional plane strain element. The element size was set to $1 \mu \mathrm{m}$ around the Si chip corner where stress concentration occurred. The materials were defined as linear elastic materials, and the values in Table 1 were used. The highest thermal stress is generated at the lowtemperature side in a TC test, because the thermal curing temperature at which the resin bonds can be regarded as stress-free and the low-temperature side has the highest temperature difference with the stress-free temperature. Therefore, thermal stress analyses were conducted with the initial temperature was $200^{\circ} \mathrm{C}$, the curing temperature of the formulations, and the package was cooled to $-55^{\circ} \mathrm{C}$.

The $\mathrm{X}$-axis direction from the $\mathrm{Si}$ chip corner was defined as the $\mathrm{H}$-interface, and the $\mathrm{Y}$-axis direction was defined as the V-interface. The relationship between the distance $(r)$ and the $\mathrm{H}$ - and V-interface stresses of the MMC4 package when the chip corner was the origin is shown in Fig. 5, where $\sigma_{\theta}$ is the normal stress, and $\tau_{r \theta}$ is the shear stress. 
$\sigma_{\theta}$ indicated compressive stress (negative), and $\tau_{r \theta}$ indicated singularity that was inversely proportional to $r$. Interfacial delamination occurs only with tensile stress and not with compressive stress. Thus, the shear stress caused the interface delamination in the TC test. Furthermore, the shear stresses of the V-interface were higher than those of the H-interface, suggesting that the V-interface was preferentially delaminated. These results were consistent with the delamination locations of the TC test shown in Fig. 3.

\subsection{Interfacial stress field in the die shear test}

The adhesive strength of the molding material is a crucial characteristic to prevent interface fracture, and one of the evaluation methods is the die shear test. Figure 6 shows a schematic of die shear testing. Si chip of specimen which composites of Si chip $(2 \mathrm{~mm} \times 2 \mathrm{~mm} \times 725 \mu \mathrm{mt}) /$ evaluation sheet $(30 \mu \mathrm{m} t) / \mathrm{Si}$ wafer $(725 \mu \mathrm{m} t)$, was pressed from the side until the Si chip detached, and the maximum strength was measured as the die shear strength. The die shear strength was measured after storage at $200^{\circ} \mathrm{C}$ for up to $200 \mathrm{~h}$ because the die shear strength is expected to change due to thermal degradation at the high-tempera-

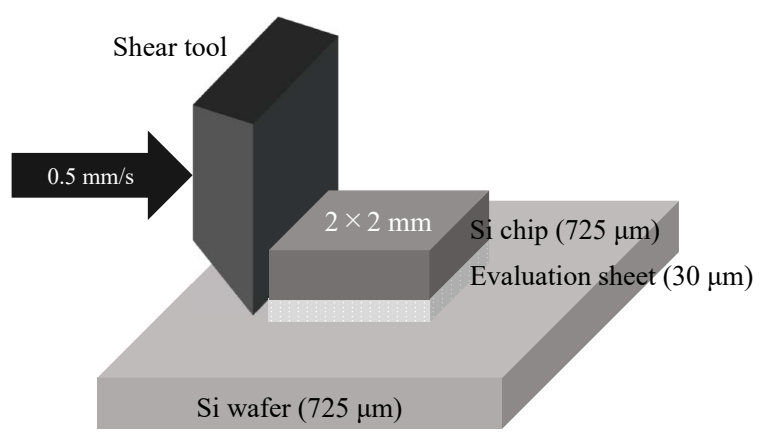

Fig. 6 Schematic depicting die shear testing.

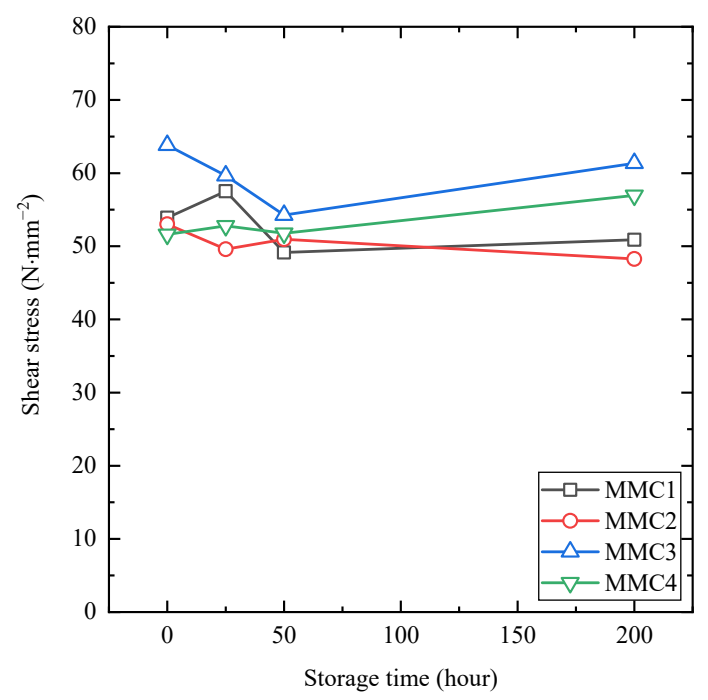

Fig. 7 Die shear stress during storage at $200^{\circ} \mathrm{C}$. ture side of the TC test, as shown in Fig. 7. Although the die shear stresses of all formulations slightly varied, they maintained high shear stress value without the degradation of adhesiveness due to thermal storage. Therefore, the die shear stress was fixed to the value before heat storage in the subsequent investigation. The stress distribution at the interface during delamination was investigated by FEM analysis using the measured shear stress values. Figure 8 presents the FEM model. The FEM element type was chosen to be a four-node two-dimensional plane strain element. The element size was set to $1 \mu \mathrm{m}$ around the pressing area of the formulation, the remaining area is divided into up to $5 \mu \mathrm{m}$. The materials were defined as linear elastic materials, and the values in Table 1 were used for their properties. The boundary conditions were set to constrain the bottom edge of the Si wafer in the X- and Y-axes opposite the shear side and the bottom edge of the wafer on the pressing side in the Y-axis. The stress distribution at the bonding interface of the formulation was analyzed when the Si chip edge was sheared at the die shear stress before thermal storage.

Figure 9 presents the relationship between the distance ( $r$ ) and the shear stress of MMC4 at the interface on the $\mathrm{Si}$ chip and Si wafer when the interface on the shear side is

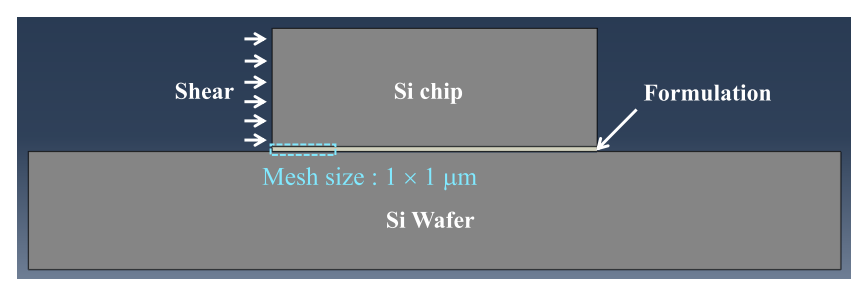

Fig. 8 FEM model of die shear testing.

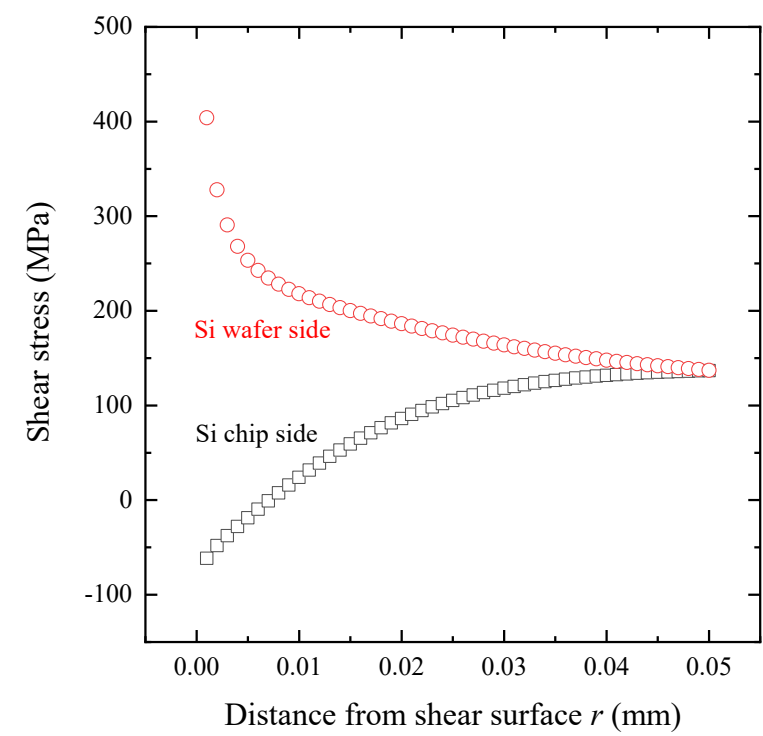

Fig. 9 Interface stress field in the die shear test of MMC4. 
the origin. The stress of the Si wafer interface was higher than that of the Si Chip interface. Thus, the interface fracture was expected to occur at the $\mathrm{Si}$ wafer side on FEM analysis. Figure 10 presents the SEM image of the Si wafer surface after the die shear test. Because a thin resin residue was observed on the wafer surface, a cohesive fracture occurred at the interface on the Si wafer side. Thus, the stress field obtained from FEM analysis was consistent with the fracture mode of the die shear test. Additionally, the stress field on the Si wafer interface increased as the approach to the press surface $(r=0)$. This meant that $r=0$ was a stress singularity point and the starting point of fracture.

\subsection{Comparison of stress singularity parameters}

The stress distribution in a singular field where the stress diverges to infinity while approaching the tip, such as the bonded interface of the package and the shear stress on the Si wafer side of the die shear test by FEM analysis, can be approximated by Eq. (1):

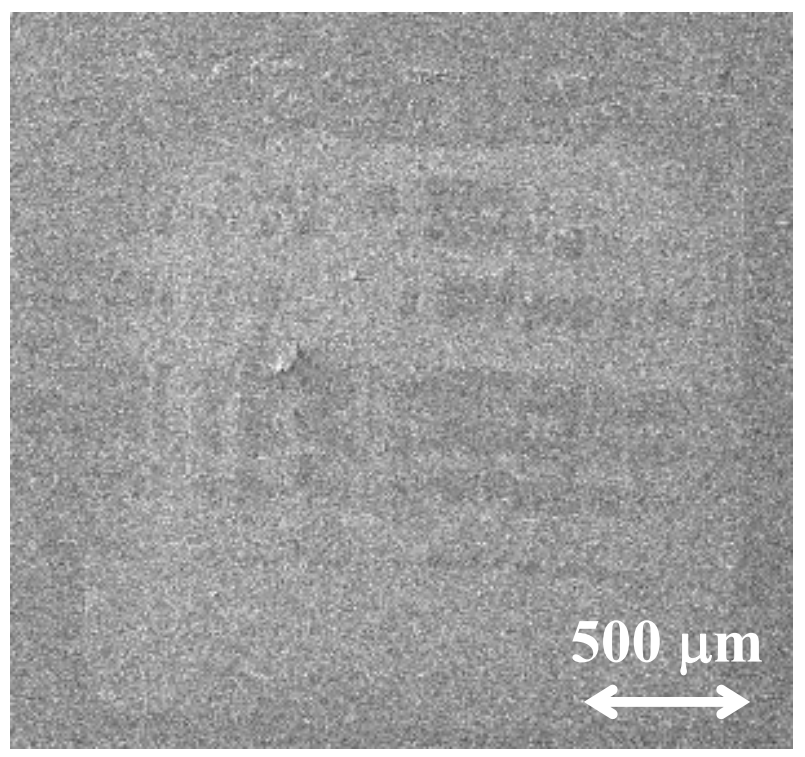

Fig. 10 SEM image of the wafer surface after the die shear test.

$$
\sigma(r)=\frac{K}{r^{\lambda}}
$$

where $\sigma, K$, and $\lambda$ are the stress, the stress singularity parameter, and the order of stress singularity, respectively. Focusing on $K$ employed as a fracture stress parameter in singular stress fields in fracture engineering, we computed and compared the $K$ values of the formulations from the interfacial stresses in the TC and die shear tests. $\lambda$ around the bonding of dissimilar materials is theorized from the bonding angle, elastic modulus, and Poisson's ratio of the materials.[18-21] However, Kitano et al. reported that $\lambda$ around the bonding interface of dissimilar materials deviates from the theoretical value.[22] Therefore, $\lambda_{\text {calc }}$ and $K_{\text {calc }}$ were determined by approximating Eq. (1) by regression analysis from the FEM stress distribution $(0<r \leq 0.02$ $\mathrm{mm}$ ) and compared with $\lambda_{\text {theory }} . \lambda_{\text {theory }}$ was computed using characteristic equatation presented in Bogy.[18, 20] For calculation of Dundurs parameter [23] in the characteristic equatation, the physical properties shown in Table 3-1 were applied.

Table 3 presents $K_{\text {calc }}^{V}, \lambda_{\text {calc }}^{V}$, and $\lambda_{\text {theory }}^{V}$ determined from the stress distribution at the V-interface of each package in the TC test, and $K_{\text {calc }}^{W}, \lambda_{\text {calc }}^{W}$, and $\lambda_{\text {theory }}^{W}$ determined from the stress distribution at the wafer side interface in the die shear test. The regression analysis value and theoretical value deviated for each condition; hence, we used $\lambda_{\text {calc }}^{V}$ and $\lambda_{\text {calc }}^{W}$ for the order of stress singularity in the following discussion. Although the values of $\lambda_{\text {calc }}^{V}$ and $\lambda_{\text {calc }}^{W}$ were different, their differences between the formulations were minor. $K_{\text {calc }}^{W}$ computed from the die shear test can serve as the fracture stress parameter of the formulations. However, $K_{\text {calc }}^{W}$ and $K_{\text {calc }}^{V}$ cannot be directly compared because they have different orders of stress singularity. Therefore, the ratio of stress singularity parameters for each condition was considered using the similarity of the order of stress singularity of the formulations. $K_{r}\left(K_{\text {calc }}^{W} / K_{\text {calc }}^{V}\right)$ decreased with inscreasing $\mathrm{MB}$ content, and $K_{r}$ of MMC1,

Table 3 Parameters and orders of stress singularity for the V-interface of the package in the TC test and the wafer interface in the die shear test.

\begin{tabular}{c|ccc|ccc|c}
\hline \multirow{2}{*}{ Formulation } & \multicolumn{3}{|c|}{$\begin{array}{c}\text { V-interface of package } \\
\text { in TC test }\end{array}$} & \multicolumn{3}{c|}{$\begin{array}{c}\text { Wafer-interface } \\
\text { in the die shear test }\end{array}$} & $\begin{array}{c}K_{r}^{W} \\
K_{\text {calc }}^{V}\end{array}$ \\
& $\begin{array}{c}K_{\text {calc }} \\
\left(\mathrm{MPa} \cdot \mathrm{mm}^{\lambda}\right)\end{array}$ & $\lambda_{\text {calc }}^{V}$ & $\lambda_{\text {theory }}^{V}$ & $\lambda_{\text {calc }}^{W}$ & $\lambda_{\text {theory }}^{W}$ & \\
\hline MMC1 & 14.0 & 0.292 & 0.398 & 66.2 & 0.256 & 0.323 & 4.73 \\
MMC2 & 22.9 & 0.291 & 0.394 & 69.2 & 0.270 & 0.321 & 3.02 \\
MMC3 & 25.8 & 0.292 & 0.409 & 69.4 & 0.268 & 0.319 & 2.69 \\
MMC4 & 31.3 & 0.293 & 0.415 & 67.5 & 0.255 & 0.317 & 2.16 \\
\hline
\end{tabular}


MMC2, MMC3, and MMC4 were 4.73, 3.02, 2.69, and 2.16 respectively. High $K_{r}$ provides strong interfacial fracture resistance. These results indicated that the reliability of the interface in the TC test decreases with the amount of MB. Especially, the interfacial fracture resistance of MMC4, which contains the highest amount of MB, was less than half that of MMC1.

\section{Crack Propagation Analysis}

\subsection{Stress field in the crack propagation direction}

Resin crack as shown in Fig. 3 is generally caused by the propagation of interfacial delamination. Then, the stress field in the crack propagation direction may vary, depending on the distance of the interfacial fracture. However, experimentally determining the relationship between interfacial fracture distance and crack propagation is difficult. Furthermore, the molding material is preferred to prevent crack propagation at any interfacial fracture distance. In this section, we proposed a method to predict crack propagation from their stress fields and fracture stresses using three interface models (Fig. 11): (a) fully bonded model, (b) micro-delaminated model, and (c) fully delaminated model. The fully bonded model is the same as the analyzed model in Section 3.1, the micro-delaminated model involves the delamination of $5 \mu \mathrm{m}$ from the Si chip corner in the direction of the V-interface, and the fully delaminated model entails the entire delamination of the V-interface. The FEM analyses of the three models were conducted to investigate the stress field in the crack propagation direction. Kitano et al. reported that the circumferential stress $\left(\sigma_{\theta}\right)$ dominates the resin crack propagating from the Si chip corner, and the parameter of stress singularity determined from the distribution of $\sigma_{\theta}$ is a reasonable stress evaluation parameter.[22] Therefore, the parameter of stress singularity $\left(K_{\theta}\right)$ and the order of stress singularity $\left(\lambda_{\theta}\right)$ in Eq. (1) were computed by the FEM at each interface delamination state in the TC test. Table 4 presents $K_{\theta}$ and $\lambda_{\theta}$ obtained from the stress of $\sigma_{\theta}$ at 135 degrees clockwise from the V-interface with the Si chip corner as the origin. Although $\lambda_{\theta}$ differed among the interface states, they were similar among the formulations. Furthermore, formulations with high MB content showed high $K_{\theta}$ and thus require a higher stress singularity criterion $\left(K_{C}\right) . K_{\theta}$ and $K_{C}$ need to be compared at a similar $\lambda_{\theta}$ as $K_{\theta}$ depends on $\lambda_{\theta}$. However, $K_{C}$ corresponding to a predetermined $\lambda_{\theta}$ is tremendously hard to obtain from the material evaluation. Therefore, we predicted the crack propagation potential from the approximate equations of $K_{\theta}$ and $\lambda_{\theta}$ obtained from FEM analysis.

The approximation equation was obtained by substituting $K_{\theta}$ and $\lambda_{\theta}$ from (a), (b), and (c) into Eq. (1), and the flexural stress criterion for crack prevention $\left(\sigma_{C}\right)$ and reference distance $\left(r_{C}\right)$ were obtained by regression analysis, as shown in Fig. 12. Table 5 presents computed $\sigma_{C}$ and $r_{C}$ for each formulation. Since $\sigma_{C}$ can be regarded as the stress criterion at $\lambda_{\theta}=0$, cracking is expected in case of

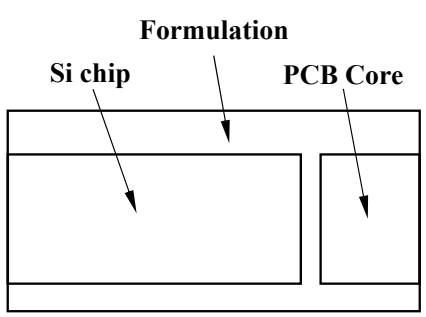

(a) Fully-bonded model

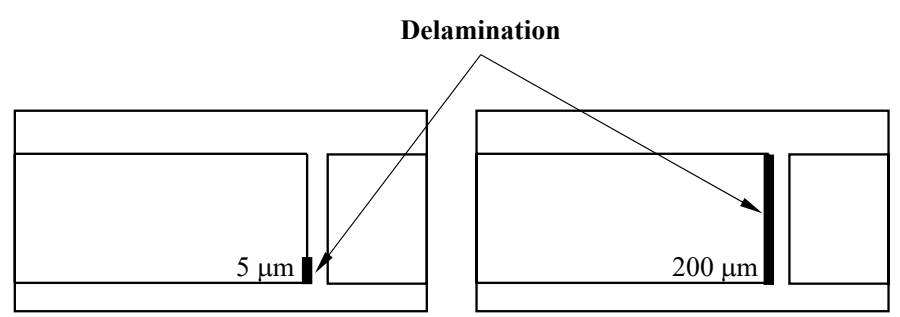

(c) Fully-delaminated model

Fig. 11 FEM model for stress analysis in the crack growth direction.

Table 4 Parameters and orders of stress singularity for each interface.

\begin{tabular}{c|cc|cc|cc}
\hline \multirow{2}{*}{ Formulation } & \multicolumn{2}{|c|}{ (a) Fully-bonded } & \multicolumn{2}{c|}{ (b) Micro-delaminatied } & \multicolumn{2}{c}{ (c) Fully-delaminated } \\
\cline { 2 - 7 } & $\lambda_{\theta}$ & $\begin{array}{c}K_{\theta} \\
\left(\mathrm{MPa} \cdot \mathrm{mm}^{\lambda}\right)\end{array}$ & $\lambda_{\theta}$ & $\begin{array}{c}K_{\theta} \\
\left(\mathrm{MPa} \cdot \mathrm{mm}^{\lambda}\right)\end{array}$ & $\lambda_{\theta}$ & $\begin{array}{c}K_{\theta} \\
\left(\mathrm{MPa} \cdot \mathrm{mm}^{\lambda}\right)\end{array}$ \\
\hline MMC1 & 0.107 & 37.5 & 0.243 & 19.6 & 0.373 & 14.8 \\
\hline MMC2 & 0.097 & 63.6 & 0.242 & 32.1 & 0.372 & 24.5 \\
\hline MMC3 & 0.097 & 71.8 & 0.241 & 36.3 & 0.371 & 27.8 \\
\hline MMC4 & 0.097 & 87.9 & 0.240 & 43.7 & 0.369 & 33.6 \\
\hline
\end{tabular}




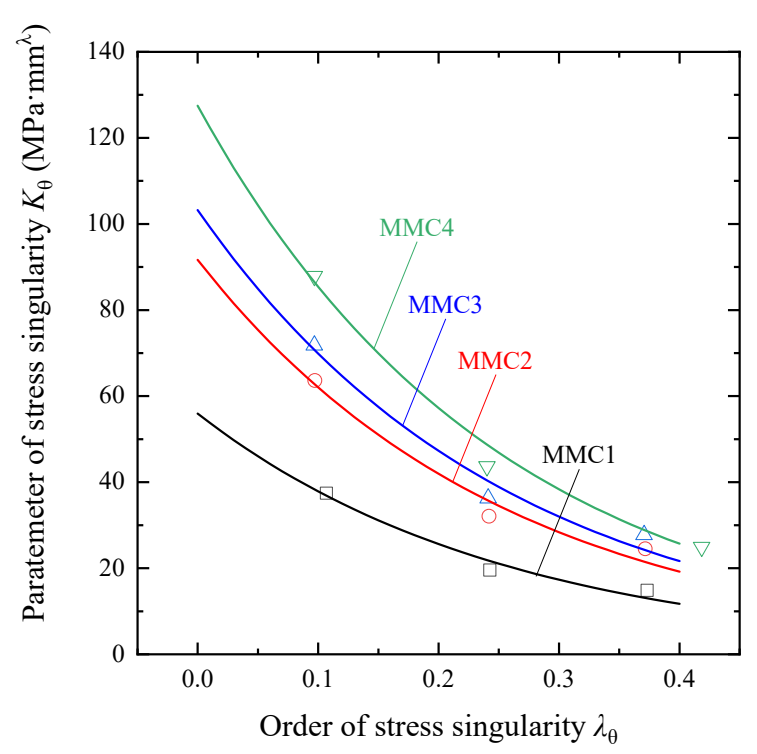

Fig. 12 Relationship between $\lambda_{\theta}$ and $K_{\theta}$ for each formulation.

Table 5 Stress criterion and reference distance for each formulation computed from $K_{\theta}$ and $\lambda_{\theta}$.

\begin{tabular}{ccc}
\hline Formulation & $\begin{array}{c}r_{C} \\
(\mathrm{~mm})\end{array}$ & $\begin{array}{c}\sigma_{C} \\
(\mathrm{MPa})\end{array}$ \\
\hline MMC1 & 0.0202 & 55.9 \\
MMC2 & 0.0201 & 91.7 \\
MMC3 & 0.0201 & 103 \\
MMC4 & 0.0183 & 127 \\
\hline
\end{tabular}

flexural stress below $\sigma_{C}$. [24]

The flexural stress $\left(\sigma_{B}\right)$ of the formulation fluctuates on the high-temperature side of the TC test. Thus, $\sigma_{B}$ was measured by three-point bending after long-term storage at $200^{\circ} \mathrm{C}$, as shown in Fig. 13. The $\sigma_{B}$ values of MMC3 and $\mathrm{MMC} 4$, where cracks occurred in the TC test, were below $\sigma_{C}$ for the storage time equivalent to 1,000 cycles (166 h), indicating the possibility of failure during the TC test. The $\sigma_{B}$ values of MMC3 and MMC4, where cracks occurred in the TC test, were below $\sigma_{C}$ for the storage time equivalent to 1,000 cycles $(166 \mathrm{~h})$, indicating the possibility of failure during the TC test. The physical properties of organic materials are generally known to degrade due to the decomposition of molecular chains in thermal storage. However, the $\sigma_{B}$ value of this formulation increased once during the thermal storage, because remaining unreactive functional groups may react to form a more robust molecular structure. In any case, since the thermal history equivalent to 1,000 cycles of the TC test promotes material degradation, the molding material must endure the changes during the test. The flexural stress $\left(\sigma_{B}\right)$ before thermal

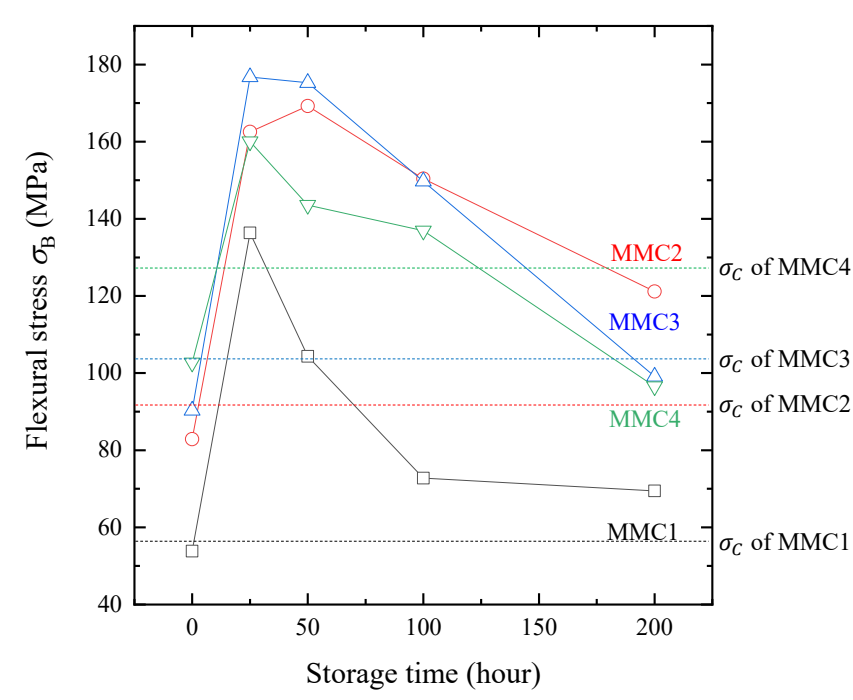

Fig. 13 Flexural stress during storage at $200^{\circ} \mathrm{C}$ and stress criterion for each material.

storage was below the stress criterion $\left(\sigma_{C}\right)$ for all formulations. We have presumed that the cracks did not occur during the storage time because the plastic deformation might have occurred due to the incomplete reaction of the formulation. To suppress resin cracking in TC tests, formulation design is required with a wide margin to the physical property variations by thermal degradation. Although the flexural stress of the material enhanced with the addition of $\mathrm{MB}$, the thermal stresses increased more than that. High flexural stress is an essential property that protects the material from physical deformation. The amount of MB must be determined by weighing the flexural stress and package reliability.

\subsection{Dependence of the stress criterion on material properties}

When a molding material is a linear elastic material, $\sigma_{C}$ in the TC test depends on the storage modulus and CTE. Here, stress analysis was conducted using the FEM to formulate a prediction equation for the stress criterion $\left(\sigma_{C}\right)$ required in the $-55^{\circ} \mathrm{C} / 200^{\circ} \mathrm{C}$ TC test based on the storage modulus and CTE of the molding material. $\sigma_{C}$ for each condition was analyzed using the procedure described in Section 4.1, where the storage modulus and CTE of the molding material were varied from 5 to $20 \mathrm{GPa}$ and from 10 to $100 \mathrm{ppm} / \mathrm{K}$, respectively. Figures. 14 and 15 present the relationship between material properties (storage modulus, CTE) and $\sigma_{C} . \sigma_{C}$ was determined to exhibit a linear relationship with the storage modulus and CTE. The relationship between $\sigma_{C}$ and CTE had a different slope depending on the storage modulus. The relationship between $\sigma_{C}$ and the storage modulus also had a different slope depending on the CTE. Thus, the storage modulus 


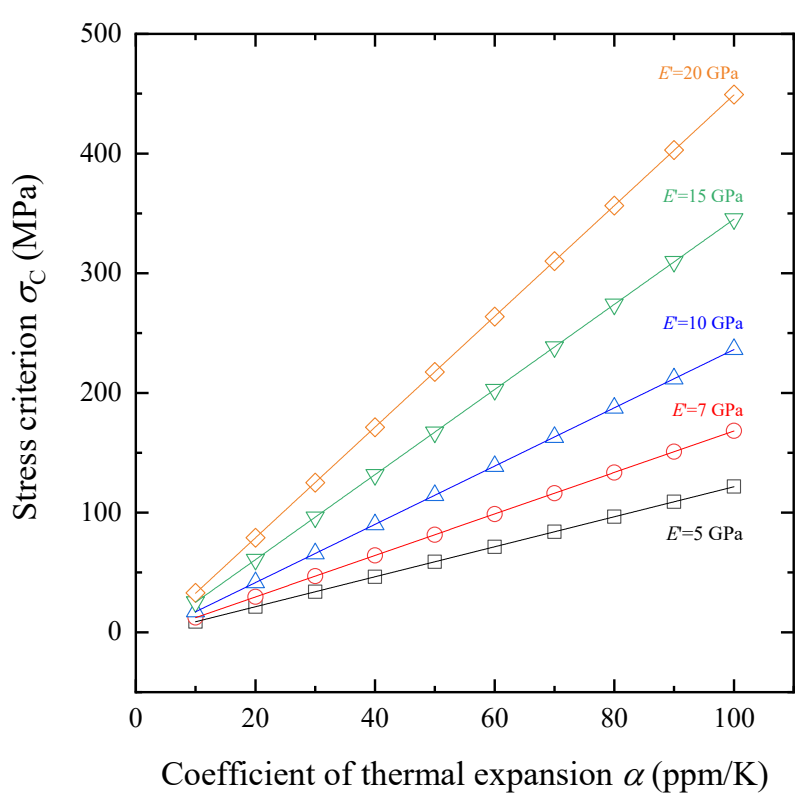

Fig. 14 CTE dependence of stress criterion for each storage modulus.

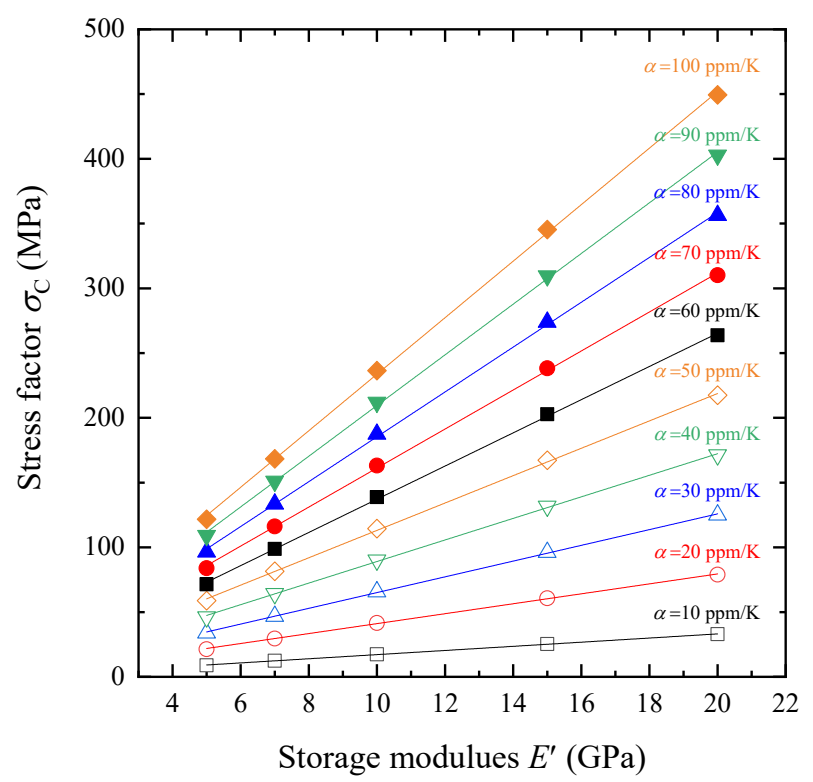

Fig. 15 Storage modulus dependence of stress criterion for each CTE.

and CTE had statistically interaction effect on $\sigma_{C}$.

From these relationships, $\sigma_{C}$ was estimated by Eq (2) using the elastic modulus (E') and CTE $(\alpha)$.

$$
\sigma_{C}=A \times\left(E^{\prime}+B\right) \times(\alpha+C)
$$

where $\mathrm{A}, \mathrm{B}$, and $\mathrm{C}$ are constants that depend on test conditions, and the regression analysis of the results in Figs. 14 and 15 gave $\mathrm{A}=0.225, \mathrm{~B}=0.705$, and $\mathrm{C}=-2.94$. $\mathrm{By}$ substituting the storage modulus and the CTE of the molding material into Eq.(2), the flexural stress required to prevent resin cracking in the TC test can be predicted.
Namely, the physical properties of the materials enable the estimation of the crack resistance of the materials, without having to fabricate an evaluation package and conduct a long-term TC test. The constant values (A, B, C) are limited to the packaging material and the temperature of the TC test, but different conditions are applicable by following the same procedure described in this section.

\section{Conclusion}

We discussed cracking and interfacial delamination in the package-level TC test of maleimide molding sheets containing flexible maleimide resin using FEM analysis and stress singularity theory. First, the interfacial stress field of the TC test package and the die shear test were investigated using FEM analysis. The stress fields were consistent with the delamination modes that occurred in the actual tests. Besides, the stress singularity parameters computed from the stress field indicated that the delamination failure was prone to occur during the TC test as the amount of the flexible maleimide resin content in the maleimide molding sheet increased. Furthermore, the flexural stress criterion for crack prevention was computed using the 2D-FEM analysis with the stress singularity theory. The maleimide molding sheet with cracks exhibited measured flexural strength below the stress criterion value, which was consistent with the TC results. The results indicated that the $\mathrm{MB}$ formulation increased the flexural stress but decreased the package reliability. Finally, a simple procedure to obtain the stress criterion from the E' and CTE of a molding material was presented. Further consideration will be needed to yield about applicability to different package structure. Since the encapsulation material was handled as a linear elastic model, the effect of using an elastoplastic or viscoelastic model should be further investigated.

\section{References}

[1] H. Lee, V. Smet, and R. Tummala, "A Review of SiC Power Module Packaging Technologies: Challenges, Advances, and Emerging Issues,” IEEE Journal of Emerging and Selected Topics in Power Electronics, Vol. 8, No. 1, pp. 239-255, 2020.

[2] A. Matallana, E. Ibarra, I. López, J. Andreu, J. Garate, $\mathrm{X}$. Jordà, and J. Rebollo, "Power module electronics in HEV/EV applications: New trends in wide-bandgap semiconductor technologies and design aspects," Renewable and Sustainable Energy Reviews, Vol. 113, Art. No. 109264, 2019. 
[3] F. Hou, W. Wang, R. Ma, Y. Li, Z. Han, M. Su, J. Li, Z. Yu, Y. Song, Q. Wang, M. Chen, L. Cao, G. Zhang, and B. Ferreira, "Fan-Out Panel-Level PCB-Embedded SiC Power MOSFETs Packaging,” IEEE Journal of Emerging and Selected Topics in Power Electronics, Vol. 8, No. 1, pp. 367-380, 2020.

[4] A. Ostmann, L. Boettcher, D. Manessis, S. Karaszkiewicz, and K.-D. Lang, "Power modules with embedded components,” 2013 Eurpoean Microelectronics Packaging Conference (EMPC), pp. 1-4, 2013.

[5] T. Loher, S. Karaszkiewicz, L. Bottcher, and A. Ostmann, "Compact power electronic modules realized by PCB embedding technology,” 2016 IEEE CPMT Symposium Japan (ICSJ), pp. 259-262, 2016.

[6] R. Khazaka, L. Mendizabal, D. Henry, and R. Hanna, "Survey of High-Temperature Reliability of Power Electronics Packaging Components,” IEEE Transactions on Power Electronics, Vol. 30, No. 5, pp. 24562464, 2015.

[7] T. Braun, S. Voges, M. Topper, M. Wilke, M. Wohrmann, U. Maas, M. Huhn, K.-F. Becker, S. Raatz, J.-U. Kim, R. Aschenbrenner, K.-D. Lang, C. Oconnor, R. Barr, J. Calvert, M. Gallagher, E. Iagodkine, T. Aoude, and A. Politis, "Material and process trends for moving from FOWLP to FOPLP," 2015 IEEE 17th Electronics Packaging and Technology Conference (EPTC), pp. 1-6, 2015.

[8] M. Guyenot, C. Mager, A. Birkhold, R. Ratchev, A. Khoshamouz, T. Gottwald, and S. Kreuer, "New Resin Materials for High Power Embedding,” 2017 IEEE 67th Electronic Components and Technology Conference (ECTC), pp. 690-695, 2017.

[9] M. Guyenot, D. Maas, R. Ratchev, A. Khoshamouz, T. Gottwald, and S. Kreuer, "New Failure Mechanism in High Temperature Resin Materials,” 2018 IEEE 68th Electronic Components and Technology Conference (ECTC), pp. 1238-1244, 2018.

[10] Y. Karasawa, K. Kikuchi, Y. Nedzu, and T. Sugino, "Application of maleimide materials for molding sheet," 24th Symposium on Microjoining and Assembly Technology in Electronics (Mate), pp. 135-138, 2018.

[11] Y. Karasawa, K. Kikuchi, T. Yoshinobu, and T. Sugino, "Molding sheet with high heat resistivity based on maleimide resin," 25th Symposium on Microjoining and Assembly Technology in Electronics (Mate), pp. 201-204, 2019.
[12] K. Kikuchi, Y. Karasawa, Y. Watanabe, T. Sugino, and T. Suetsugu, "Improvement of Brittleness for Maleimide Molding Film and Package Level Reliability,” 2019 IEEE CPMT Symposium Japan (ICSJ), pp. 177-181, 2019.

[13] K. Kikuchi, Y. Nedzu, and T. Sugino, "Warpage Analysis with Newly Molding Material of Fan-Out Panel Level Packaging and the Board Level Reliability Test Results,” 2018 IEEE 68th Electronic Components and Technology Conference (ECTC), pp. 973-978, 2018.

[14] K. Kikuchi, Y. Nezu, and T. Sugino, "Sheet-Shape Molding Resin Realizing Low Warpage for Fan-Out Panel Level Package,” Journal of Institute of Electronics, Information and Communication Engineers, Vol. J101-C, No. 7, pp. 273-281, 2018.

[15] K. Xue, J. Wu, H. Chen, Y. Sun, K. Kwan, J. Yuen, and A. Lam, "Numerical analysis of interfacial delamination in thin array plastic package,” 2008 International Conference on Electronic Packaging Technology \& High Density Packaging, pp. 1-3, 2008.

[16] R. Harries and S. Sitaraman, "Numerical modeling of interfacial delamination propagation in a novel peripheral array package,” IEEE Transactions on Components and Packaging Technologies, Vol. 24, No. 2, pp. 256-264, 2001.

[17] C. L. Gall, J. Qu, and D. Mcdowell, "Delamination cracking in encapsulated flip chips,” 1996 Proceedings 46th Electronic Components and Technology Conference (ECTC), pp. 430-434, 1996.

[18] D. B. Bogy, "Two Edge-Bonded Elastic Wedges of Different Materials and Wedge Angles Under Surface Tractions,” Journal of Applied Mechanics, Vol. 38, No. 2, pp. 377-386, 1971.

[19] P. S. Theocaris and E. E. Gdoutos, "Stress singularities in cracked composite full-planes," International Journal of Fracture, Vol. 13, No. 6, pp. 763-773, 1977.

[20] D. Bogy and K. Wang, "Stress singularities at interface corners in bonded dissimilar isotropic elastic materials," International Journal of Solids and Structures, Vol. 7, No. 8, pp. 993-1005, 1971.

[21] J. C. W. Vroonhoven, "Stress Singularities In BiMaterial Wedges With Adhesion And Delamination," Fatigue \& Fracture of Engineering Materials and Structures, Vol. 15, No. 2, pp. 159-171, 1992.

[22] M. Kitano, A. Nishimura, and S. Kawai, "A Study of Package Cracking during the Reflow Soldering Pro- 
cess (2nd Report, Strength Evaluation of the Plastic by Using Stress Singularity Theory)," Transactions of the Japan Society of Mechanical Engineers, Series A, Vol. 57, No. 538, pp. 1387-1405, 1991.

[23] J. Dundurs, "Discussion: 'Edge-bonded dissimilar orthogonal elastic wedges under normal and shear loading' (Bogy, D. B., 1968, ASME J. Appl. Mech., 35, pp. 460-466),” Journal of Applied Mechanics, Vol. 36, No. 3, pp. 650-652, 1969.

[24] T. Hattori, S. Sakata, and G. Murakami, "A Stress Singularity Parameter Approach for Evaluating the Interfacial Reliability of Plastic Encapsulated LSI Devices,” Journal of Electronic Packaging, Vol. 111, No. 4, pp. 243-248, 1989.

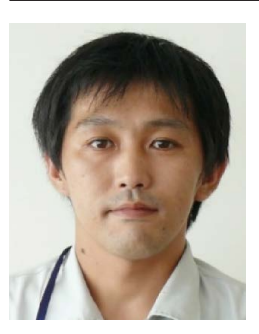

Kazuhiro Kikuchi received the B. E. degree from the University of Electro-Communications, Tokyo, Japan, in 2005. He joined Lintec Corporation and has been engaged in the research and development of advanced semiconductor packaging processes and materials.

Kazue Uemura received the M. E. degree from Niigata University, Niigata, Japan, in 2007. She joined Lintec Corporation in 2007 and has been engaged in the research and development of adhesive materials.

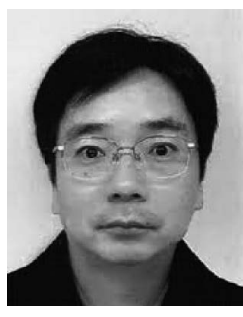

Tadashi Suetsugu received the B. E., M. $\mathrm{E}$., and $\mathrm{Ph}$. D. degrees in electrical engineering from the Department of Electrical Engineering, Keio University, Yokohama, Japan, in 1990, 1992, and 1995, respectively.

He was an Assistant Professor from 1995 to 1998 with the Department of Electronics, Fukuoka University, Fukuoka, Japan. In 2001-2002, he was a Visiting Professor with the Department of Electrical Engineering, Wright State University, Dayton, OH, USA. Since 1998, he has been with the Department of Electronics Engineering and Computer Science, Fukuoka University, where he is currently a Professor and director of 3D Semiconductor Research Center in Fukuoka. His research interests include semiconductor packaging, high-frequency high-efficiency switching-mode tuned power amplifiers, resonant $\mathrm{dc} / \mathrm{dc}$ power converters, dc/ac inverters, high-frequency rectifiers, numerical simulation of switching circuits, and power line communications. He is senior member of IEEE, member of IEICE, IEEJ. 